\title{
Ineffective perturbations in a planar elastica
}

\author{
Kaitlyn Peterson and Robert Manning
}

(Communicated by Natalia Hritonenko)

\begin{abstract}
An elastica is a bendable one-dimensional continuum, or idealized elastic rod. If such a rod is subjected to compression while its ends are constrained to remain tangent to a single straight line, buckling can occur: the elastic material gives way at a certain point, snapping to a lower-energy configuration.

The bifurcation diagram for the buckling of a planar elastica under a load $\lambda$ is made up of a trivial branch of unbuckled configurations for all $\lambda$ and a sequence of branches of buckled configurations that are connected to the trivial branch at pitchfork bifurcation points. We use several perturbation expansions to determine how this diagram perturbs with the addition of a small intrinsic shape in the elastica, focusing in particular on the effect near the bifurcation points.

We find that for almost all intrinsic shapes $\varepsilon f(s)$, the difference between the buckled solution and the trivial solution is $O\left(\varepsilon^{1 / 3}\right)$, but for some ineffective $f$, this difference is $O(\varepsilon)$, and we find functions $u_{j}(s)$ so that $f$ is ineffective at bifurcation point number $j$ when $\left\langle f, u_{j}\right\rangle=0$. These ineffective perturbations have important consequences in numerical simulations, in that the perturbed bifurcation diagram has sharper corners near the former bifurcation points, and there is a higher risk of a numerical simulation inadvertently hopping between branches near these corners.
\end{abstract}

\section{Introduction}

Consider a common scenario for symmetry breaking in bifurcation theory. A problem exhibiting some symmetry has a bifurcation diagram with a number of bifurcation points (BPs). The addition of a perturbation breaks this symmetry and removes the BPs, splitting the diagram into separate components. An example of this scenario is shown in Figure 1, in which a pitchfork bifurcation is perturbed to yield two separate branches.

MSC2000: 34B15, 34E10, 34G99, 74K10.

Keywords: elastic rod, intrinsic shape, undetermined-gauges perturbation expansion, pitchfork bifurcations.

This work was supported by NSF grant DMS-0384739. 

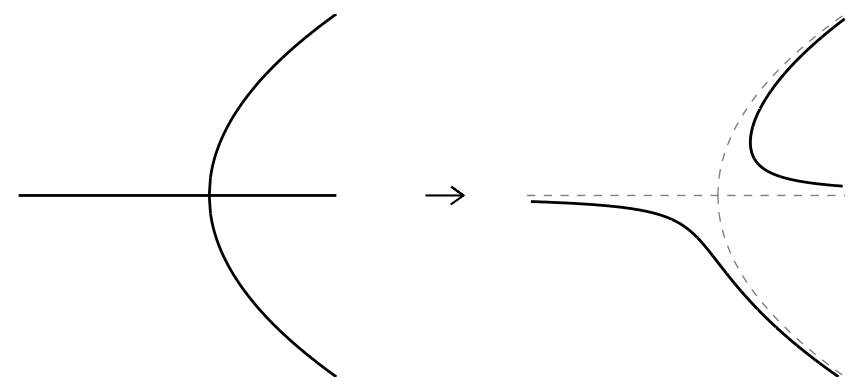

Figure 1. Standard perturbation of a pitchfork bifurcation into two separate branches.

To give a specific example, the buckling of a uniform, isotropic, intrinsically straight rod in three dimensions has a bifurcation diagram containing pitchfork BPs corresponding to the classic Euler buckling modes. There is a natural perturbation to consider for this three-dimensional buckling problem: the presence of intrinsic curvature. Even rods designed to be straight are likely to have small curvature imperfections, and these can break the qualitative nature of the bifurcation diagram from the pitchfork structure seen in the intrinsically straight case.

Such elastic rod models have been used to represent the bending and twisting of DNA. For many DNA sequences, the intrinsic shape is nearly straight, but the minimum-energy stacking configurations of consecutive base-pairs do introduce small intrinsic bends that depend on the specific sequence of the DNA. Multiple studies have sought to determine these stacking configurations as a function of sequence [De Santis et al. 1992; Bolshoy et al. 1991; Olson et al. 1998; Dixit et al. 2005], and then derive from these stacking configurations the corresponding intrinsic curvature for a continuum elastic rod [Manning et al. 1996].

These DNA models have seen increasing use in studying a phenomenon called DNA looping: the bending and twisting of DNA a few hundreds of base-pairs long in response to prescribed relative positions and orientations of the two ends (these boundary conditions coming from, for example, a bound protein of known structure [Swigon et al. 2006; Goyal et al. 2007; Kahn and Crothers 1998] or laser tweezer experiments [Seol et al. 2007; Marko and Siggia 1995]). Given the wide variety of DNA sequences, and the almost-as-wide variety of parameters for determining local bending from sequence, it would be beneficial to have an automated algorithm to compute the lowest-energy components of the bifurcation diagram given specified choices of DNA sequence, stacking parameters, and boundary conditions. A strong understanding of the splitting of the unperturbed pitchfork diagram for base cases such as buckling or periodic boundary conditions is an important precursor to ensuring that such an automated algorithm finds all relevant components of the diagram. 
How typical is the "standard" splitting shown in Figure 1? We analyzed this question for one of the simplest bifurcation problems - the buckling of a planar elastica - under the family of perturbations of (infinitesimal) intrinsic curvature. Like the three-dimensional buckling problem described above, this problem exhibits a sequence of pitchfork BPs. This two-dimensional problem is useful as a base case for the more general three-dimensional DNA looping problem. The formulation of two-dimensional bending is simple enough that closed-form analysis is not too unwieldy; at the same time, it seems reasonable to suppose that the threedimensional results would be analogous, since, in some sense, three-dimensional bending is composed of two orthogonal directions of two-dimensional bending. The particular buckling boundary conditions we choose yield a classic problem in mechanical engineering, but are not the typical boundary conditions for DNA looping. Still, a likely computational approach to determining configurations obeying arbitrary DNA looping boundary conditions would involve beginning from one of a small number of simple configurations, one of which would be the straight-rod configuration studied here (in addition, to, perhaps, a circle and a semicircle).

Thus, this choice of a simple model problem should allow us to focus on the fundamental questions of which perturbations are atypical and how the bifurcation diagrams of atypical perturbations differ from the typical case. Our main finding is that for these atypical cases, the perturbation of the BP is significantly smaller than in the typical case, leading us to label these perturbations as ineffective.

This question is related to the analysis of unfolding a pitchfork bifurcation diagram in dynamical systems; see [Glendinning 1994; Iooss and Joseph 1980]. The standard example is to consider the algebraic equation $\lambda x-x^{3}=0$, which exhibits a pitchfork BP at $\lambda=0$. The addition of a second parameter, for example, $\alpha+\lambda x-x^{3}=0$, splits the diagram as in Figure 1 if $\alpha \neq 0$. The boundary case $\alpha=0$ is the analogue of the ineffectiveness condition we derive for the elastica, although the analysis and final result are more involved since our mathematical setting is an ODE (plus boundary conditions and an integral constraint) rather than an algebraic equation, and the perturbations considered are a space of functions rather than a single parameter. Furthermore, in the standard unfolding study, the focus is generally on $\alpha=0$ as the transition between qualitative behaviors, whereas we focus on determining the leading-order behaviors of solutions both away from, and directly on, this boundary case.

In addition to this theoretical analysis, we present some computational results motivated by the idea of deriving an automated algorithm to determine DNA looping configurations. One approach to performing such computations is to use exactly the symmetry-breaking path considered here: begin with the symmetric problem for which solutions are known, and proceed via a continuation algorithm to numerical solutions for the perturbed problem. We explore how these continuation 
algorithms can fail if the perturbation is ineffective (or even nearly ineffective), due to the presence of sharp corners in a branch of solutions. Thus, in designing a system for automatically computing such bifurcation diagrams for a wide range of intrinsic shapes, the ineffectivity conditions we derive serve as an important red flag that numerical difficulties may arise in a specific subset of computations.

Our analysis proceeds as follows. First, we formulate the planar buckling problem in Section 2, including an $O(\varepsilon)$ intrinsic-curvature term. Next, in Section 3, we apply a standard perturbation expansion to the trivial branch of $\varepsilon=0$ unbuckled configurations. Away from the BPs, this expansion gives an $O(\varepsilon)$ approximation to the perturbation of the trivial branch. At the BPs, this analysis breaks down for most intrinsic curvature profiles, but for certain special profiles, it does still yield an $O(\varepsilon)$ solution. These cases are exactly the ineffective perturbations, and we derive conditions for when they occur. In Section 4, we apply an alternative perturbation technique called undetermined gauges to the effective perturbations at the BPs and find an $O\left(\varepsilon^{1 / 3}\right)$ leading-order term for the perturbation of the unbuckled configuration. Finally, in Section 5, we present several examples illustrating the theory and a computational study verifying the numerical difficulties created by ineffective or nearly ineffective perturbations.

\section{The planar buckling problem}

We consider an inextensible and unshearable elastic rod in the plane, assumed for simplicity to have total arc-length 1 . We parametrize the rod by arc-length $s$, and denote the configuration of the rod at arc-length-value $s$ by $(x(s), y(s))$. We choose coordinates and boundary conditions as shown in Figure 2: the $s=0$ end of the rod is at the origin, we impose clamped boundary conditions at each end requiring the

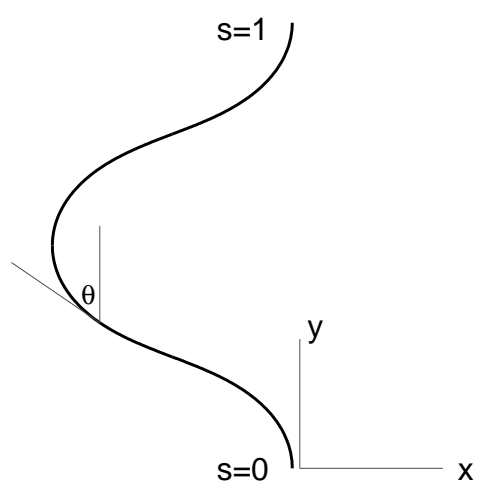

Figure 2. Boundary conditions on the planar elastica. The $s=0$ end of the rod is held at the origin with vertical tangent, while the $s=1$ end of the rod is held at $x=0$, also with vertical tangent. 
tangent vectors to be vertical, and further require $x(1)=0$. The inextensibilityunshearability constraint implies that $\left(x^{\prime}(s), y^{\prime}(s)\right)$ is a unit vector; this allows us to describe the rod by a single unknown function $\theta(s)$, where $\left(x^{\prime}(s), y^{\prime}(s)\right)=$ $(\cos \theta(s), \sin \theta(s))$. The clamped boundary conditions are given by $\theta(0)=\theta(1)=$ 0 , and the additional constraint $x(1)=0$ can be rewritten as $\int_{0}^{1} \sin \theta(s) d s=0$.

We place a mass $m>0$ at the $s=1$ end of the rod, and assume the following functional for the energy of the rod-plus-mass system:

$$
E[\theta] \equiv \int_{0}^{1}\left(\frac{K}{2}\left(\theta^{\prime}(s)-\varepsilon f(s)\right)^{2}+m g \cos \theta(s)\right) d s .
$$

The first term represents the bending energy of the rod, and the second term the potential energy of the load. The term $\varepsilon f(s)$ is used to model intrinsic curvature: the minimum-energy configuration of the rod has $\theta^{\prime}(s)=\varepsilon f(s)$, and deviations from $\varepsilon f(s)$ involve a quadratic energy cost, weighted by the stiffness parameter $K$. For simplicity, we express energy in units of $K$, and define $\lambda=m g / K>0$, so that the energy functional becomes

$$
E[\theta]=\int_{0}^{1}\left(\frac{1}{2}\left(\theta^{\prime}(s)-\varepsilon f(s)\right)^{2}+\lambda \cos \theta(s)\right) d s .
$$

We thus consider the calculus of variations problem to find critical points of $E$ subject to the boundary conditions $\theta(0)=\theta(1)=0$ and the isoperimetric constraint $\int_{0}^{1} \sin \theta(s) d s=0$. These critical points are found by solving the ordinary differential equation (ODE) defined by the Euler-Lagrange equation (with Lagrange multiplier $\mu$ included because of the isoperimetric constraint):

$$
\theta^{\prime \prime}(s)=\varepsilon f^{\prime}(s)-\lambda \sin \theta(s)+\mu \cos \theta(s) .
$$

Thus, the mathematical problem we considered was, given known values for the load $\lambda$, perturbation parameter $\varepsilon$, and intrinsic curvature profile $f(s)$ (with $f^{\prime}$ not identically zero, since otherwise $\varepsilon$ has no effect), find solutions $(\theta(s), \mu)$ of

$$
\begin{gathered}
\theta^{\prime \prime}(s)=\varepsilon f^{\prime}(s)-\lambda \sin \theta(s)+\mu \cos \theta(s), \\
\theta(0)=\theta(1)=0, \quad \int_{0}^{1} \sin \theta(s) d s=0 .
\end{gathered}
$$

For $\varepsilon=0$, the solutions to (1) as $\lambda$ varies yield the familiar force-length diagram seen in Figure 3. (All bifurcation diagrams in this article were computed using the parameter-continuation package AUTO97 [Doedel et al. 1991a; 1991b].) One solution (for each value of $\lambda$ ) is $\theta(s) \equiv 0, \mu=0$ (a straight rod), and this corresponds to the horizontal line at the top of Figure 3. We call this the trivial branch. 


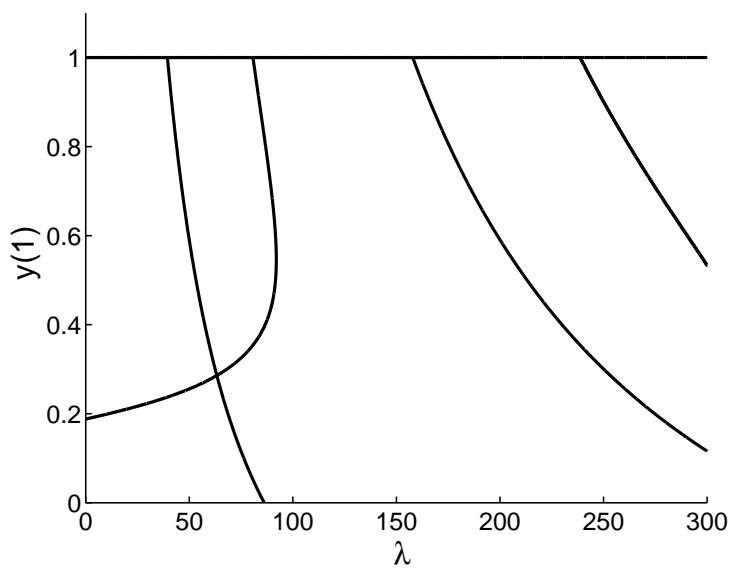

Figure 3. Bifurcation diagram for a planar elastica with no intrinsic curvature $(\varepsilon=0)$. The height of the top of the $\operatorname{rod}(y(1))$ is plotted against the imposed load $\lambda$.

There are pitchfork bifurcation points ${ }^{1}$ at all values of $\lambda$ satisfying

$$
2-2 \cos \sqrt{\lambda}-\sqrt{\lambda} \sin \sqrt{\lambda}=0
$$

(see Section 3 for a derivation of this equation). This equation has a countable sequence of solutions that we will label as $0<\lambda_{1}<\lambda_{2}<\cdots$. For $n$ odd,

$$
\lambda_{n}=(n+1)^{2} \pi^{2},
$$

whereas for $n$ even,

$$
(n+0.5)^{2} \pi^{2}<\lambda_{n}<(n+1)^{2} \pi^{2}, \quad \text { with }(n+1)^{2} \pi^{2}-\lambda_{n} \rightarrow 0 \quad \text { as } n \rightarrow \infty .
$$

Two properties of $\lambda_{n}$ (for $n$ even) will be useful to us:

Lemma 1. For $n$ even,

$$
\sin \sqrt{\lambda_{n}}=\frac{4 \sqrt{\lambda_{n}}}{\lambda_{n}+4}, \quad \cos \sqrt{\lambda_{n}}=\frac{4-\lambda_{n}}{\lambda_{n}+4} .
$$

Proof. By (2), we have

$$
1-\cos \sqrt{\lambda_{n}}=\frac{\sqrt{\lambda_{n}} \sin \sqrt{\lambda_{n}}}{2}, \quad 1+\cos \sqrt{\lambda_{n}}=2-\frac{\sqrt{\lambda_{n}} \sin \sqrt{\lambda_{n}}}{2} .
$$

\footnotetext{
${ }^{1}$ The appearance of the pitchfork bifurcation points in Figure 3 differs slightly from the standard picture from Figure 1 since the two outer prongs of the pitchfork are folded on top of each other due to the choice of $y(1)$ as ordinate.
} 
Multiplying these two equations, we find

$$
\sin ^{2} \sqrt{\lambda_{n}}=\sqrt{\lambda_{n}} \sin \sqrt{\lambda_{n}}-\frac{\lambda_{n} \sin ^{2} \sqrt{\lambda_{n}}}{4} .
$$

Collecting terms,

$$
\sin ^{2} \sqrt{\lambda_{n}}\left(1+\frac{\lambda_{n}}{4}\right)=\sqrt{\lambda_{n}} \sin \sqrt{\lambda_{n}},
$$

and since $\sin \sqrt{\lambda_{n}} \neq 0$ for $n$ even, we may divide both sides by it and solve for $\sin \sqrt{\lambda_{n}}$ to find the desired result.

The formula for $\cos \sqrt{\lambda_{n}}$ follows from the formula

$$
\cos \sqrt{\lambda_{n}}=-\sqrt{1-\sin ^{2} \sqrt{\lambda_{n}}}
$$

(note the minus sign due to the fact that $\sqrt{\lambda_{n}}$ is just below an odd multiple of $\pi$ ).

Lemma 2. For $n$ even, $-\lambda_{n}-\lambda_{n} \cos \sqrt{\lambda_{n}}+2 \sqrt{\lambda_{n}} \sin \sqrt{\lambda_{n}}=0$.

Proof. Since $(n+0.5)^{2} \pi^{2}<\lambda_{n}<(n+1)^{2} \pi^{2}$, we have

$$
\sin \sqrt{\lambda_{n}} \neq 0 \quad \text { and } \quad 1-\cos \left(\sqrt{\lambda_{n}}\right) \neq 0 .
$$

Thus

$$
\frac{\sin \sqrt{\lambda_{n}}}{1-\cos \sqrt{\lambda_{n}}}=\frac{1+\cos \sqrt{\lambda_{n}}}{\sin \sqrt{\lambda_{n}}},
$$

since cross-multiplying yields the identity $1-\cos ^{2} \sqrt{\lambda_{n}}=\sin ^{2} \sqrt{\lambda_{n}}$. By (2), the left side of (3) equals $2 / \sqrt{\lambda_{n}}$, and therefore,

$$
\frac{1+\cos \sqrt{\lambda_{n}}}{\sin \sqrt{\lambda_{n}}}=\frac{2}{\sqrt{\lambda_{n}}}
$$

Cross-multiplying, and multiplying both sides by $\sqrt{\lambda_{n}}$, yields the desired equality.

\section{Perturbation of trivial branch for small $\varepsilon$}

For fixed $\lambda>0, f(s)$, and $\varepsilon>0$, we now seek a solution to (1) that will be close to the solution on the trivial branch for $\varepsilon=0$. We use a standard perturbation analysis, writing

$$
\theta(s)=\theta_{0}(s)+\varepsilon \theta_{1}(s)+\cdots, \quad \mu=\mu_{0}+\varepsilon \mu_{1}+\cdots .
$$

The $O(1)$ terms $\theta_{0}(s)$ and $\mu_{0}$ vanish since $\mu=0$ and $\theta(s) \equiv 0$ on the trivial branch. Therefore, we seek the $O(\varepsilon)$ terms $\theta_{1}(s)$ and $\mu_{1}$, by plugging these expansions into (1). After Taylor expanding $\sin \theta$ and $\cos \theta$ and isolating the $O(\varepsilon)$ terms we find

$$
\theta_{1}^{\prime \prime}=f^{\prime}(s)-\lambda \theta_{1}+\mu_{1}, \quad \theta_{1}(0)=\theta_{1}(1)=0, \quad \int_{0}^{1} \theta_{1}(s) d s=0 .
$$


By a standard integrating factor approach, we find the general solution to the ODE in (4):

$$
\theta_{1}(s)=\frac{1}{\sqrt{\lambda}} \int_{0}^{s} f^{\prime}(t) \sin (\sqrt{\lambda}(s-t)) d t+\frac{\mu_{1}}{\lambda}+C_{1} \cos (\sqrt{\lambda} s)+C_{2} \sin (\sqrt{\lambda} s)
$$

When we require that this general solution satisfy the remaining conditions in (4), we find three linear equations in $\mu_{1}, C_{1}$, and $C_{2}$ :

$$
\begin{aligned}
{\left[\begin{array}{ccc}
1 / \lambda & 1 & 0 \\
1 / \lambda & \cos \sqrt{\lambda} & \sin \sqrt{\lambda} \\
1 / \lambda & (\sin \sqrt{\lambda}) / \sqrt{\lambda} & (1-\cos \sqrt{\lambda}) / \sqrt{\lambda}
\end{array}\right]\left[\begin{array}{l}
\mu_{1} \\
C_{1} \\
C_{2}
\end{array}\right] } \\
=\left[\begin{array}{c}
(1 / \sqrt{\lambda}) \int_{0}^{1} f^{\prime}(t) \sin (\sqrt{\lambda}(1-t)) d t \\
(1 / \sqrt{\lambda}) \int_{0}^{1} \int_{0}^{s} f^{\prime}(t) \sin (\sqrt{\lambda}(s-t)) d t d s
\end{array}\right]
\end{aligned}
$$

The bottom term on the right side can be simplified by switching the order of integration:

$$
\int_{0}^{1} \int_{t}^{1} \frac{f^{\prime}(t)}{\sqrt{\lambda}} \sin (\sqrt{\lambda}(s-t)) d s d t
$$

and then computing the inner integral:

$$
\frac{1}{\lambda} \int_{0}^{1} f^{\prime}(t)[1-\cos (\sqrt{\lambda}(1-t))] d t .
$$

Inserting this into (5), and multiplying both sides by $\lambda$, gives

$$
\left[\begin{array}{ccc}
1 & \lambda & 0 \\
1 & \lambda \cos \sqrt{\lambda} & \lambda \sin \sqrt{\lambda} \\
1 & \sqrt{\lambda} \sin \sqrt{\lambda} & \sqrt{\lambda}(1-\cos \sqrt{\lambda})
\end{array}\right]\left[\begin{array}{l}
\mu_{1} \\
C_{1} \\
C_{2}
\end{array}\right]=\left[\begin{array}{c}
0 \\
\sqrt{\lambda} \int_{0}^{1} f^{\prime}(t) \sin (\sqrt{\lambda}(1-t)) d t \\
\int_{0}^{1} f^{\prime}(t)[1-\cos (\sqrt{\lambda}(1-t))] d t
\end{array}\right]
$$

The matrix on the left has determinant $\lambda^{3 / 2}(2 \cos \sqrt{\lambda}-2+\sqrt{\lambda} \sin \sqrt{\lambda})$, so (6) has a unique solution if $2 \cos \sqrt{\lambda}-2+\sqrt{\lambda} \sin \sqrt{\lambda} \neq 0$. In other words, referring back to (2), we have shown that away from the bifurcation points, that is, for $\lambda \neq \lambda_{n}$, the standard perturbation expansion yields a solution, that is, a $O(\varepsilon)$ approximation to $\theta(s)$ near the trivial branch.

(We note that the BP condition (2) can be derived by a computation much like the one just completed; in that instance, we would be looking for solutions near the trivial branch to the problem without the $f^{\prime}$ term. We would use the same perturbation expansion, resulting in (6) but with a zero vector as the right side, and so we would have a nontrivial solution (a BP) exactly when the determinant of the matrix vanishes.) 
What is particularly interesting for our study is whether (6) might have a solution even at a bifurcation point. Here we can use a fact from linear algebra (see [Shifrin and Adams 2002, Section 3.4], for example):

Theorem 1. For an $n \times n$ matrix $A$, the column space of $A$ is equal to the orthogonal complement of the null-space of $A^{T}$.

Denote by $\boldsymbol{b}$ the right-hand side of (6). We want to know whether $\boldsymbol{b}$ is in the column space of the matrix $\boldsymbol{A}$ on the left side, which by Theorem 1 is true if and only if $\langle\boldsymbol{b}, \boldsymbol{u}\rangle=0$ for all null-vectors $\boldsymbol{u}$ of $\boldsymbol{A}^{T}$. The null-vector for $\lambda=\lambda_{n}$ has a different form depending on whether $n$ is odd or even.

If $n$ is odd, then $\lambda_{n}=(n+1)^{2} \pi^{2}$ and

$$
\boldsymbol{A}^{T}=\left[\begin{array}{ccc}
1 & 1 & 1 \\
(n+1)^{2} \pi^{2} & (n+1)^{2} \pi^{2} & 0 \\
0 & 0 & 0
\end{array}\right],
$$

so the null space of $\boldsymbol{A}^{T}$ is span $\{(-1,1,0)\}$.

If $n$ is even, no obvious simplification can be made to the form of the matrix:

$$
\boldsymbol{A}^{T}=\left[\begin{array}{ccc}
1 & 1 & 1 \\
\lambda & \cos \sqrt{\lambda} & \sqrt{\lambda} \sin \sqrt{\lambda} \\
0 & \lambda \sin \sqrt{\lambda} & \sqrt{\lambda}(1-\cos \sqrt{\lambda})
\end{array}\right],
$$

but, using (2) and Lemma 2, one sees that the null-space of $\boldsymbol{A}^{T}$ is span $\{(-1,-1,2)\}$.

Combining these null-vectors of $\boldsymbol{A}^{T}$ with Theorem 1 shows that (6) has a solution at $\lambda_{n}$ if and only if

$$
\begin{aligned}
& \int_{0}^{1} f^{\prime}(t) \sin \left(\sqrt{\lambda_{n}}(1-t)\right) d t=0 \text { for } n \text { odd, } \\
& \int_{0}^{1} f^{\prime}(t)\left[2-2 \cos \left(\sqrt{\lambda_{n}}(1-t)\right)-\sqrt{\lambda_{n}} \sin \left(\sqrt{\lambda_{n}}(1-t)\right)\right] d t=0 \text { for } n \text { even. }
\end{aligned}
$$

Intrinsic curvature profiles $f$ satisfying (7) for some $n$ are the ineffective perturbations for bifurcation point $\lambda_{n}$.

\section{Undetermined-gauges analysis of bifurcation points}

We now turn to the analysis of the bifurcation points $\lambda=\lambda_{n}$. In Section 3 we showed that for the ineffective perturbations defined by (7), the standard perturbation analysis predicts an $O(\varepsilon)$ lowest-order term for $\theta_{1}$ and $\mu_{1}$, but that this analysis fails for the remaining perturbations. Here we investigate those cases by applying a more general technique, the methods of undetermined gauges [Murdock 1999], which is used to derive the leading-order behavior when it does not follow the standard $O(\varepsilon)$ pattern. 
We are, as before, considering (1), but now we formulate a more general perturbation expansion for $\theta$ and $\mu$. This expansion is computed one term at a time, so we begin by writing

$$
\theta(s)=\delta_{1}(\varepsilon) \theta_{1}(s), \quad \mu=\delta_{1}(\varepsilon) \mu_{1},
$$

where $\delta_{1}(\varepsilon)$ is an unknown function of $\varepsilon$ that our analysis will determine. We will restrict attention to the family $\delta_{1}(\varepsilon)=\varepsilon^{a}$ for $a$ real. We insert these expressions into (1) and expand the sin and cos as Taylor series:

$$
\begin{aligned}
& \delta_{1} \theta_{1}^{\prime \prime}=\varepsilon f^{\prime}(s)-\lambda_{n}\left(\delta_{1} \theta_{1}+\cdots\right)+\left(\delta_{1} \mu_{1}\right)(1+\cdots), \\
& \delta_{1} \theta_{1}(0)=\delta_{1} \theta_{1}(1)=0, \quad \int_{0}^{1}\left(\delta_{1} \theta_{1}+\cdots\right) d s=0 .
\end{aligned}
$$

We want to look at the leading order terms, but in the ODE, there is a question of whether $\delta_{1}$ or $\varepsilon$ is dominant, or if they could be of the same order. If $\varepsilon$ were dominant, then the leading-order terms of the ODE would be the nonsensical $0=$ $\varepsilon f^{\prime}(s)$. If $\delta_{1}$ and $\varepsilon$ were of the same order, that is, $\delta_{1}=\varepsilon$, then the leading-order terms of the ODE would give the same equation as in the standard perturbation expansion, which we know from Section 3 has no solution. Hence, the dominant term must be $\delta_{1}$, so we keep the $O\left(\delta_{1}\right)$ terms to find

$$
\theta_{1}^{\prime \prime}=-\lambda_{n} \theta_{1}+\mu_{1}, \quad \theta_{1}(0)=\theta_{1}(1)=0, \quad \int_{0}^{1} \theta_{1}(s) d s=0 .
$$

The general solution of the ODE is $\theta_{1}(s)=C_{1} \cos \left(\sqrt{\lambda_{n}} s\right)+C_{2} \sin \left(\sqrt{\lambda_{n}} s\right)+\mu_{1} / \lambda_{n}$. Imposing $\theta_{1}(0)=0$, we find $\mu_{1}=-\lambda_{n} C_{1}$, and then the other two conditions give the linear system

$$
\left[\begin{array}{lc}
\cos \sqrt{\lambda_{n}}-1 & \sin \sqrt{\lambda_{n}} \\
\frac{\sin \sqrt{\lambda_{n}}}{\sqrt{\lambda_{n}}}-1 & \frac{1-\cos \sqrt{\lambda_{n}}}{\sqrt{\lambda_{n}}}
\end{array}\right]\left[\begin{array}{l}
C_{1} \\
C_{2}
\end{array}\right]=\left[\begin{array}{l}
0 \\
0
\end{array}\right] .
$$

Since the determinant of the matrix in this system is zero by (2), we have nontrivial solutions $\left(C_{1}, C_{2}\right)$, namely any nonzero null-vector of the matrix. If $n$ is odd, the matrix simplifies to $\left[\begin{array}{rr}0 & 0 \\ -1 & 0\end{array}\right]$, so $(0,1)$ is a null-vector and the solution coming from this first gauge — still with $n$ odd - is

$$
\begin{gathered}
\theta_{1}=C \sin \left(\sqrt{\lambda_{n}} s\right)=C \sin (\pi(n+1) s) \quad(C \neq 0 \text { to be determined }), \\
\mu_{1}=0 .
\end{gathered}
$$


On the other hand, if $n$ is even, then $\left(\sin \sqrt{\lambda_{n}}, 1-\cos \sqrt{\lambda_{n}}\right)$ is a null-vector of the matrix, which means that the solution coming from this first gauge is

$$
\begin{aligned}
& \theta_{1}=k\left[\sin \sqrt{\lambda_{n}}\left(\cos \left(\sqrt{\lambda_{n}} s\right)-1\right)+\left(1-\cos \sqrt{\lambda_{n}}\right) \sin \left(\sqrt{\lambda_{n}} s\right)\right], \\
& \mu_{1}=-k \lambda_{n} \sin \sqrt{\lambda_{n}} .
\end{aligned}
$$

By (2), $1-\cos \sqrt{\lambda_{n}}=\frac{1}{2} \sqrt{\lambda_{n}} \sin \sqrt{\lambda_{n}}$, so that this solution can be rewritten as

$$
\theta_{1}=k \sin \sqrt{\lambda_{n}}\left(\cos \left(\sqrt{\lambda_{n}} s\right)-1+\frac{1}{2} \sqrt{\lambda_{n}} \sin \left(\sqrt{\lambda_{n}} s\right)\right), \quad \mu_{1}=-k \lambda_{n} \sin \sqrt{\lambda_{n}} .
$$

For simplicity, we write $k \sin \sqrt{\lambda_{n}} / 2$ as a single constant $C$ for the final form of the solution for $n$ even:

$$
\begin{gathered}
\theta_{1}=C\left(2 \cos \left(\sqrt{\lambda_{n}} s\right)-2+\sqrt{\lambda_{n}} \sin \left(\sqrt{\lambda_{n}} s\right)\right) \quad(C \neq 0 \text { to be determined }), \\
\mu_{1}=-2 C \lambda_{n} .
\end{gathered}
$$

In order to determine $C$ and $\delta_{1}$, we add another gauge.

$$
\theta=\delta_{1}(\varepsilon) \theta_{1}(s)+\delta_{2}(\varepsilon) \theta_{2}(s), \quad \mu=\delta_{1}(\varepsilon) \mu_{1}+\delta_{2}(\varepsilon) \mu_{2},
$$

for $\delta_{2}(\varepsilon)$ an unknown function of $\varepsilon$ (again in the family $\varepsilon^{a}$ ), by definition of lower order in $\varepsilon$ than $\delta_{1}$. As before, we insert these expressions into (1) and Taylor-expand the sin and cos terms, looking for the next-lowest-order terms after $O\left(\delta_{1}\right)$.

For the boundary conditions, these next-lowest-order terms give $\delta_{2}(\varepsilon) \theta_{2}(0)=$ $\delta_{2}(\varepsilon) \theta_{2}(1)=0$, or $\theta_{2}(0)=\theta_{2}(1)=0$. As for the integral condition, since $\sin \theta=$ $\theta-\theta^{3} / 6+\cdots$, there are two next-lowest-order candidates: $\delta_{2}$ from the $\theta$ term, and $\delta_{1}^{3}$ from the $\theta^{3}$ term. We list them both for the time being:

$$
\int_{0}^{1}\left(\delta_{2} \theta_{2}(s)-\frac{1}{6}\left(\delta_{1}\right)^{3} \theta_{1}(s)^{3}+\cdots\right) d s=0,
$$

Finally, we look at the ODE. The sin term yields the same two possible secondlowest-order terms $\delta_{2}$ or $\left(\delta_{1}\right)^{3}$ as in the integral condition, and so, in fact, does the cos term ( $\delta_{2}$ from the $\delta_{2} \mu_{2}$ term in $\mu$ times the 1 in the cos expansion, or $\left(\delta_{1}\right)^{3}$ from the $\delta_{1} \mu_{1}$ term in $\mu$ times the $-\left(\delta_{1} \theta_{1}\right)^{2} / 2$ from the cos expansion):

$$
\begin{aligned}
\delta_{2} \theta_{2}^{\prime \prime}=\varepsilon f^{\prime}(s)-\lambda_{n}\left[\delta_{2} \theta_{2}(s)-\frac{1}{6}\left(\delta_{1}\right)^{3}\left(\theta_{1}(s)\right)^{3}+\cdots\right] & \\
+ & {\left[\delta_{2} \mu_{2}-\frac{1}{2}\left(\delta_{1}\right)^{3} \mu_{1}\left(\theta_{1}(s)\right)^{2}+\cdots\right] . }
\end{aligned}
$$

Overall, there are three candidates for next-lowest-order term: $\varepsilon, \delta_{2}$, and $\left(\delta_{1}\right)^{3}$. We have to consider all possibilities for the relative rankings of these terms, including ties. The arguments below rule out all possibilities except having all three of the same order. 
Case 1: $\varepsilon$ lowest-order. This cannot be true, since the dominant terms in (11) would give $0=f^{\prime}(s)$, but by assumption $f^{\prime}$ is not identically zero.

Case 2: $\left(\delta_{1}\right)^{3}$ lowest-order. This cannot be true, since the dominant terms in (11) would give

$$
0=-\frac{1}{6}\left(\theta_{1}(s)\right)^{2}\left(\theta_{1}(s)+3 \mu_{1}\right),
$$

and neither $\theta_{1}$ nor $\theta_{1}+3 \mu_{1}$ is identically zero.

Case 3: $\delta_{2}$ lowest-order. The dominant terms in (11) would give the same equation we solved for $\theta_{1}$ and $\mu_{1}$ (including the boundary and integral conditions), so that $\theta_{2}=\theta_{1}$ and $\mu_{2}=\mu_{1}$. Thus, our gauge expansions would reduce to $\theta=\left(\delta_{1}+\delta_{2}\right) \theta_{1}$ and $\mu=\left(\delta_{1}+\delta_{2}\right) \mu_{1}$, and we would essentially be back where we began this step, having replaced the unknown $\delta_{1}(\varepsilon)$ by another unknown $\delta_{1}(\varepsilon)+\delta_{2}(\varepsilon)$, without having learned anything about the connection of $\delta_{1}$ to $\varepsilon$. Thus, we reject this case.

Case 4: $\varepsilon$ and $\left(\delta_{1}\right)^{3}$ tied for lowest-order. Since $\left(\delta_{1}\right)^{3}=\varepsilon$, the dominant terms in (11) would give $0=f^{\prime}(s)-\frac{1}{6}\left(\theta_{1}(s)\right)^{3}$. This requires the perturbation $f^{\prime}$ to take the very particular form of the cubes of the functions (8) or (9). Since this case does not yield a solution for a general perturbation, we reject this case.

Case 5: $\varepsilon$ and $\delta_{2}$ tied for lowest-order. This cannot be true: we would have $\delta_{2}=\varepsilon$ and the dominant terms in (11) would give the same equation (4) from the standard perturbation expansion, as well as the same integral and boundary conditions, and we know that this system has no solution for $\lambda=\lambda_{n}$ and the effective perturbations we are considering in this section.

Case 6: $\delta_{2}$ and $\left(\delta_{1}\right)^{3}$ tied for lowest order. We have $\left(\delta_{1}\right)^{3}=\delta_{2}$ and the dominant terms in (11) would give

$$
\theta_{2}^{\prime \prime}=-\lambda_{n} \theta_{2}+\frac{1}{6} \lambda_{n}\left(\theta_{1}\right)^{3}+\mu_{2}-\frac{1}{2} \mu_{1}\left(\theta_{1}\right)^{2} .
$$

This equation can be solved in closed form using the forms of $\mu_{1}$ and $\theta_{1}$ from (8) and (9), as follows.

For $n$ odd, we have $\mu_{1}=0$ and $\theta_{1}=C \sin \left(\sqrt{\lambda_{n}} s\right)$, and the ODE has solution

$$
\begin{aligned}
\theta_{2}(s)=C_{1} \cos \left(\sqrt{\lambda_{n}} s\right)+ & C_{2} \sin \left(\sqrt{\lambda_{n}} s\right) \\
& +\mu_{2} / \lambda_{n}-\frac{1}{16} C^{3} s \sqrt{\lambda_{n}} \cos \left(\sqrt{\lambda_{n}} s\right)+\frac{1}{192} C^{3} \sin \left(3 \sqrt{\lambda_{n}} s\right) .
\end{aligned}
$$

Applying $\theta_{2}(0)=\theta_{2}(1)=0$ leads to the impossible conclusion that $C=0$.

For $n$ even, the solution of the ODE is

$$
\begin{aligned}
\theta_{2}(s)=C_{1} \cos \left(\sqrt{\lambda_{n}} s\right)+C_{2} & \sin \left(\sqrt{\lambda_{n}} s\right)+\mu_{2} / \lambda_{n}+\frac{8}{3} C^{3} \\
+\frac{1}{16} C^{3}\left(\lambda_{n}-12\right)(2- & \left.\lambda_{n} s\right) \cos \left(\sqrt{\lambda_{n}} s\right)+\frac{1}{96} C^{3}\left(3 \lambda_{n}-4\right) \cos \left(3 \sqrt{\lambda_{n}} s\right) \\
+ & \frac{1}{192} C^{3} \sqrt{\lambda_{n}}\left(\lambda_{n}-12\right)\left[24 s \sin \left(\sqrt{\lambda_{n}} s\right)+\sin \left(3 \sqrt{\lambda_{n}} s\right)\right] .
\end{aligned}
$$


The condition $\theta_{2}(0)=0$ allows us to solve for $\mu_{2}$, leaving

$$
\begin{array}{r}
\theta_{2}(s)=C_{1}\left[\cos \left(\sqrt{\lambda_{n}} s\right)-1\right]+C_{2} \sin \left(\sqrt{\lambda_{n}} s\right)+\frac{1}{96} C^{3}\left(148-15 \lambda_{n}\right) \\
+\frac{1}{16} C^{3}\left(\lambda_{n}-12\right)\left(2-\lambda_{n} s\right) \cos \left(\sqrt{\lambda_{n}} s\right)+\frac{1}{96} C^{3}\left(3 \lambda_{n}-4\right) \cos \left(3 \sqrt{\lambda_{n}} s\right) \\
+\frac{1}{192} C^{3} \sqrt{\lambda_{n}}\left(\lambda_{n}-12\right)\left[24 s \sin \left(\sqrt{\lambda_{n}} s\right)+\sin \left(3 \sqrt{\lambda_{n}} s\right)\right] .
\end{array}
$$

Next we impose the boundary condition $\theta_{2}(1)=0$, and using Lemma 1, we find

$$
\theta_{2}(1)=-\frac{2 \lambda_{n}}{4+\lambda_{n}} C_{1}+\frac{4 \sqrt{\lambda_{n}}}{4+\lambda_{n}} C_{2}+\frac{C^{3} \lambda_{n}^{2}\left(\lambda_{n}-12\right)}{16\left(4+\lambda_{n}\right)}=0 .
$$

Recalling (10), since $\delta_{2}=\left(\delta_{1}\right)^{3}$, the integral condition is

$$
\int_{0}^{1}\left(\theta_{2}(s)-\frac{1}{6}\left(\theta_{1}(s)\right)^{3}\right) d s=0 .
$$

Again using Lemma 1, we can simplify this to

$$
-\frac{\lambda_{n}}{4+\lambda_{n}} C_{1}+\frac{2 \sqrt{\lambda_{n}}}{4+\lambda_{n}} C_{2}+\frac{C^{3} \lambda_{n}\left(20+\lambda_{n}\right)}{8\left(4+\lambda_{n}\right)}=0 .
$$

Subtracting two times (14) from (13) gives

$$
\frac{C^{3} \lambda_{n}^{2}\left(\lambda_{n}-12\right)}{16\left(4+\lambda_{n}\right)}-\frac{C^{3} \lambda_{n}\left(20+\lambda_{n}\right)}{4\left(4+\lambda_{n}\right)}=\frac{C^{3} \lambda_{n}\left(\lambda_{n}-20\right)}{16}=0,
$$

which implies either $C=0, \lambda_{n}=0$, or $\lambda_{n}=20$, none of which is true.

Having ruled out all other cases, we can conclude that $\delta_{2},\left(\delta_{1}\right)^{3}$ and $\varepsilon$ are all of the same order, that is, $\delta_{1}=\varepsilon^{1 / 3}$ and $\delta_{2}=\varepsilon$. In particular, we have shown that $\theta=O\left(\varepsilon^{1 / 3}\right)$.

In fact, this second gauge allows us to completely determine the leading-order behavior of $\theta(s)$ and $\mu$, as summarized by the following theorem:

Theorem 2. For $n$ odd, we have $\theta(s)=C \varepsilon^{1 / 3} \sin \left(\sqrt{\lambda_{n}} s\right)+\cdots$ and $\mu=\varepsilon \mu_{2}+\cdots$, with $\lambda_{n}=\pi(n+1)$,

$$
C=\left(\frac{16}{\lambda_{n}} \int_{0}^{1} f^{\prime}(t) \sin \left(\sqrt{\lambda_{n}}(1-t)\right) d t\right)^{1 / 3}, \mu_{2}=-\int_{0}^{1} f^{\prime}(t)\left[1-\cos \left(\sqrt{\lambda_{n}}(1-t)\right)\right] d t .
$$

For $n$ even, we have $\theta(s)=C \varepsilon^{1 / 3}\left(2 \cos \left(\sqrt{\lambda_{n}} s\right)-2+\sqrt{\lambda_{n}} \sin \left(\sqrt{\lambda_{n}} s\right)\right)+\cdots$ and $\mu=-2 \varepsilon^{1 / 3} C \lambda_{n}+\cdots$, with

$$
C=\left(\frac{16}{\left(\lambda_{n}\right)^{2}\left(\lambda_{n}-20\right)} \int_{0}^{1} f^{\prime}(t)\left[2-2 \cos \left(\sqrt{\lambda_{n}}(1-t)\right)-\sqrt{\lambda_{n}} \sin \left(\sqrt{\lambda_{n}}(1-t)\right)\right] d t\right)^{1 / 3} .
$$


Proof. For $n$ even, all that remains is to determine $C$, while for $n$ odd, we must compute $C$ and $\mu_{2}$. The derivation largely follows the computations in Case 6 . The ODE to be solved is

$$
\theta_{2}^{\prime \prime}=f^{\prime}-\lambda_{n} \theta_{2}+\frac{1}{6} \lambda_{n}\left(\theta_{1}\right)^{3}+\mu_{2}-\frac{1}{2} \mu_{1}\left(\theta_{1}\right)^{2}
$$

identical to Case 6 except for the addition of $f^{\prime}$ to the right side. Therefore, the solution of the ODE will be the expression found in Case 6 plus the term

$$
\frac{1}{\sqrt{\lambda_{n}}} \int_{0}^{s} f^{\prime}(t) \sin \left(\sqrt{\lambda_{n}}(s-t)\right) d t
$$

the solution to the equation $\theta_{2}^{\prime \prime}=f^{\prime}-\lambda_{n} \theta_{2}$ seen in Section 3. Since this new term vanishes at $s=0$, it will have no effect on the first step from Case 6 (in which we get an expression for $\mu_{2}$ using the condition $\left.\theta_{2}(0)=0\right)$.

Thus, for $n$ odd, the solution to (15) plus $\theta_{2}(0)=0$ is

$$
\begin{aligned}
\theta_{2}(s)=C_{1}\left(\cos \left(\sqrt{\lambda_{n}} s\right)\right. & -1)+C_{2} \sin \left(\sqrt{\lambda_{n}} s\right)-\frac{1}{16} C^{3} s \sqrt{\lambda_{n}} \cos \left(\sqrt{\lambda_{n}} s\right) \\
& +\frac{1}{192} C^{3} \sin \left(3 \sqrt{\lambda_{n}} s\right)+\frac{1}{\sqrt{\lambda_{n}}} \int_{0}^{s} f^{\prime}(t) \sin \left(\sqrt{\lambda_{n}}(s-t)\right) d t .
\end{aligned}
$$

Next we impose the condition $\theta_{2}(1)=0$ to find the given formula for $C$. Note that the integral does not vanish (and hence $C \neq 0$ as required) since that is our definition of what makes a perturbation $f^{\prime}$ effective.

Finally, we impose the condition

$$
\int_{0}^{1} \theta_{2}(s) d s=0
$$

(for $n$ odd, the quantity $\left(\theta_{1}\right)^{3}=\sin ^{3}(\pi(n+1) s$ ) has zero integral, so this term drops out of the integral condition) to find

$$
C_{1}=\frac{1}{\sqrt{\lambda_{n}}} \int_{0}^{1} \int_{0}^{s} f^{\prime}(t) \sin \left(\sqrt{\lambda_{n}}(s-t)\right) d t d s=\frac{1}{\lambda_{n}} \int_{0}^{1} f^{\prime}(t)\left[1-\cos \left(\sqrt{\lambda_{n}}(1-t)\right)\right] d t,
$$

where the second equality comes from switching the order of integration as in Section 3. Since $\mu_{2}=-C_{1} \lambda_{n}$ (from the $\theta_{2}(0)$ condition), we find the given formula for $\mu_{2}$.

For $n$ even, the solution to (15) plus $\theta_{2}(0)=0$ is (12) plus the term (16). Using the same steps as in Case 6 , the boundary condition $\theta_{2}(1)=0$ and the integral 
condition yield the equations

$-\frac{2 \lambda_{n}}{4+\lambda_{n}} C_{1}+\frac{4 \sqrt{\lambda_{n}}}{4+\lambda_{n}} C_{2}+\frac{C^{3} \lambda_{n}^{2}\left(\lambda_{n}-12\right)}{16\left(4+\lambda_{n}\right)}+\frac{1}{\sqrt{\lambda_{n}}} \int_{0}^{1} f^{\prime}(t) \sin \left(\sqrt{\lambda_{n}}(1-t)\right) d t=0$, $-\frac{\lambda_{n}}{4+\lambda_{n}} C_{1}+\frac{2 \sqrt{\lambda_{n}}}{4+\lambda_{n}} C_{2}+\frac{C^{3} \lambda_{n}\left(20+\lambda_{n}\right)}{8\left(4+\lambda_{n}\right)}+\frac{1}{\lambda_{n}} \int_{0}^{1} f^{\prime}(t)\left(1-\cos \left(\sqrt{\lambda_{n}}(1-t)\right)\right) d t=0$.

Subtracting twice the second equation from the first yields the formula for $C$.

\section{Examples}

Bifurcation diagrams with effective and ineffective perturbations. We consider four perturbations $f^{\prime}$ :

$f_{1}^{\prime}$, effective for both BPs,

$f_{2}^{\prime}$, ineffective for the first BP and effective for the second,

$f_{3}^{\prime}$, effective for the first BP and ineffective for the second,

$f_{4}^{\prime}$, ineffective for both BPs.

Specifically, we first define

$$
\begin{aligned}
& u_{1}(s)=\sqrt{2} \sin \left(\sqrt{\lambda_{1}}(1-s)\right), \\
& u_{2}(s)=\frac{1}{\sqrt{\lambda_{2}}}\left[2-2 \cos \left(\sqrt{\lambda_{2}}(1-s)\right)-\sqrt{\lambda_{2}} \sin \left(\sqrt{\lambda_{2}}(1-s)\right)\right] .
\end{aligned}
$$

These are length-1 elements in $L^{2}$ in the directions of the functions that define ineffectiveness for the BPs, in the sense that $f^{\prime}$ is ineffective at the $n$-th BP if $\left\langle f^{\prime}, u_{n}\right\rangle=0$. We note that $u_{1}$ and $u_{2}$ are orthogonal.

We define our four perturbations by

$$
\begin{aligned}
& f_{1}^{\prime}=\left(u_{1}+u_{2}\right) / \sqrt{2}, \quad f_{2}^{\prime}=u_{2}, \quad f_{3}^{\prime}=u_{1}, \\
& f_{4}^{\prime}(s)=\sqrt{2 \pi^{2}-3}(s+\sin (2 \pi s) / \pi) /(\pi \sqrt{6}) .
\end{aligned}
$$

By design, all the $f_{j}^{\prime}$ have length 1 (to allow comparisons); $f_{2}^{\prime}$ is orthogonal to $u_{1}$, $f_{3}^{\prime}$ is orthogonal to $u_{2}, f_{4}^{\prime}$ is orthogonal to both $u_{1}$ and $u_{2}$; and all other pairings of $f_{j}^{\prime}$ with $u_{k}$ are not close to orthogonal.

The bifurcation diagrams for these perturbations, with $\varepsilon=1$, are shown in the four panels of Figure 4. The difference between effective and ineffective perturbations is clear: in each case where a perturbation is effective, the diagram is relatively smooth near the former BP, whereas in the ineffective cases, the diagram has a sharp corner. Indeed, to numerically compute some of these corners required a significant amount of care, for example, the use of a very small step size, or a temporary increase in $\varepsilon$ by an order of magnitude just to get onto the perturbation of the bifurcating branch. 

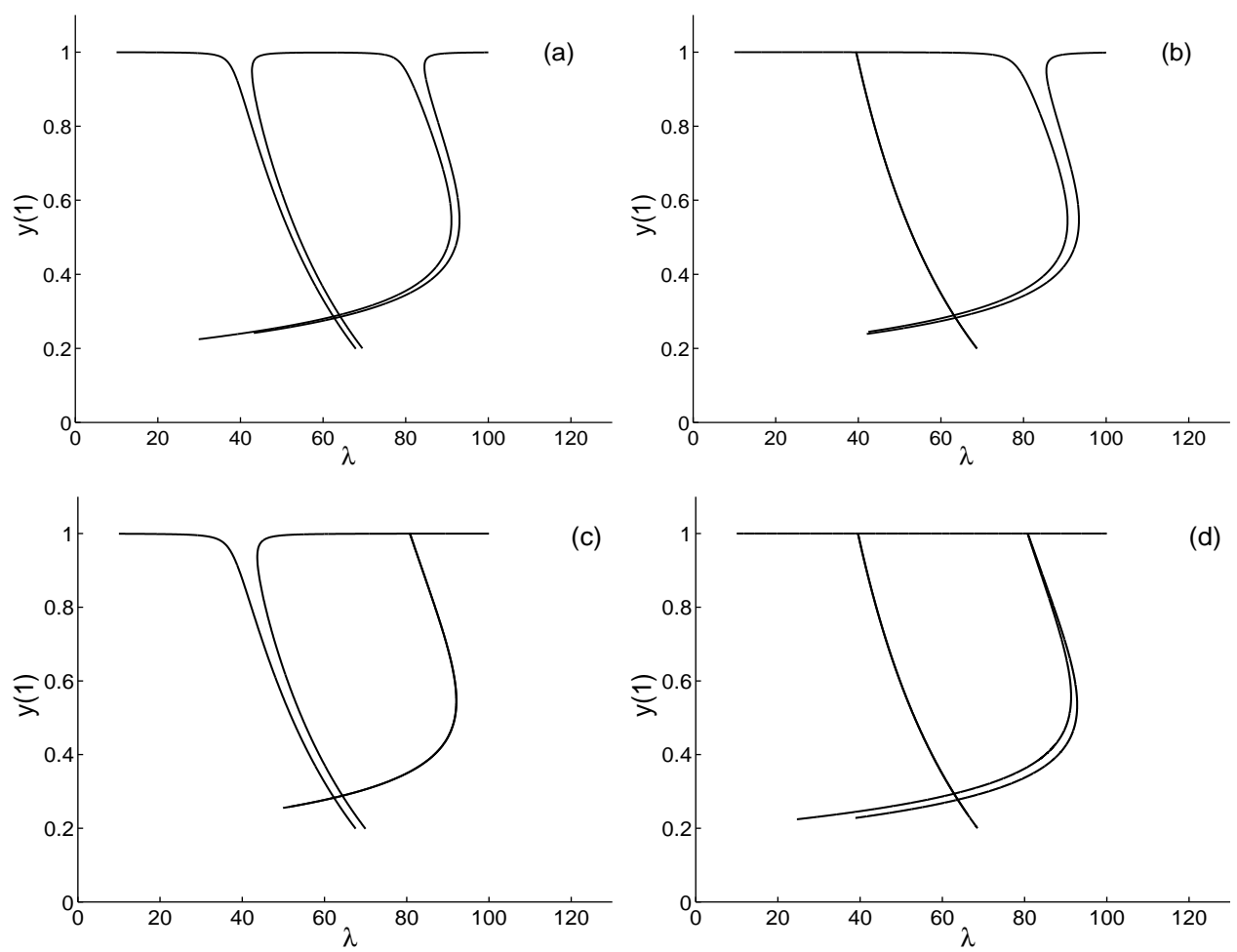

Figure 4. Bifurcation diagrams for elastica perturbed by intrinsic curvature profiles $f^{\prime}$ (see previous page for the specific functions used): (a) effective perturbation at both BPs; (b) ineffective at the first BP, effective at the second; (c) effective at the first BP, ineffective at the second; (d) ineffective at both BPs.

Leading-order behaviors in $\boldsymbol{\varepsilon}$. Finally, we show two examples illustrating the lowest-order expressions for $\theta(s)$ and $\mu$ found in Section 4. In our first example, We take $f^{\prime}(s)=s$, a perturbation that is effective at $\lambda=\lambda_{1}\left(\left\langle f^{\prime}, u_{1}\right\rangle=0.39\right)$ and ineffective at $\lambda=\lambda_{2}\left(\left\langle f^{\prime}, u_{2}\right\rangle=0\right)$. In Figure 5, we show the graphs of $\theta(s)$ for $\lambda=\lambda_{1}, \lambda_{2}$, and for $\varepsilon=\frac{1}{4}, \frac{1}{2}$, and 1 .

Our theoretical prediction for the behavior at $\lambda=\lambda_{1}$ comes from Theorem 2 (since $f^{\prime}$ is effective). We compute $\lambda_{1}=2 \pi$,

$$
C=\left(\frac{16}{4 \pi^{2}} \int_{0}^{1} t \sin (2 \pi(1-t)) d t\right)^{1 / 3}=0.401
$$

and

$$
\mu_{2}=-\int_{0}^{1} t(1-\cos (2 \pi(1-t))) d t=-0.5
$$



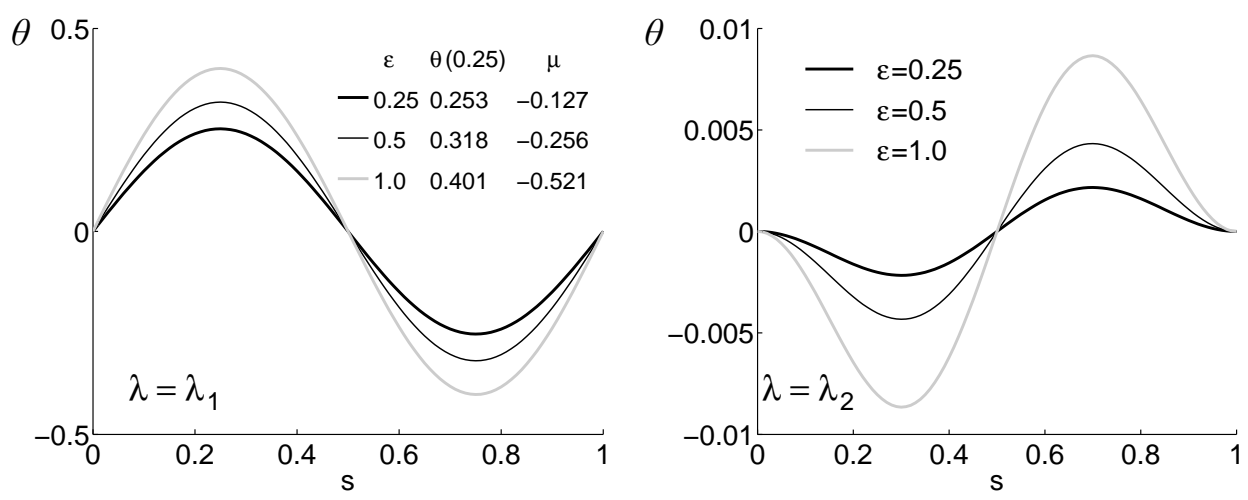

Figure 5. Graphs of $\theta(s)$ for $f^{\prime}(s)=s, \lambda=\lambda_{1}, \lambda_{2}$ and $\varepsilon=\frac{1}{4}$, $\frac{1}{2}, 1$. For $\lambda=\lambda_{1}$, the dependence of $\theta$ on $\varepsilon$ seems to be larger than $O(\varepsilon)$, in line with the $O\left(\varepsilon^{1 / 3}\right)$ prediction of the theory. The values of $\theta\left(\frac{1}{4}\right)$ (the maxima) and $\mu$ are also reported. For $\lambda=\lambda_{2}$, the dependence of $\theta$ on $\varepsilon$ appears to be approximately $O(\varepsilon)$, in line with the fact that $f^{\prime}$ is ineffective at $\lambda_{2}$.

Thus, our predicted behavior at $\lambda=\lambda_{1}$ is

$$
\theta(s) \approx 0.401 \varepsilon^{1 / 3} \sin (2 \pi s), \quad \mu \approx-0.5 \varepsilon .
$$

The shape of the actual solution $\theta(s)$ in Figure 5 matches the predicted $\sin (2 \pi s)$, and the scaling with $\varepsilon$ is clearly larger than $O(\varepsilon)$. Furthermore, from the table inset in the figure, we see that both $\theta\left(\frac{1}{4}\right)$ (the heights of the peaks) and $\mu$ are close matches with our predicted formulas.

As for $\lambda=\lambda_{2}$, since $f^{\prime}$ satisfies the ineffectivity condition, we expect $\theta(s)$ to have $O(\varepsilon)$ behavior rather than $O\left(\varepsilon^{1 / 3}\right)$. Indeed, we see in Figure 5 that this appears to be the case, as $\theta(s)$ appears to be roughly halved when $\varepsilon$ is halved. In this case, our theory does not give a predictive formula for the leading-order behavior of $\theta(s)$ or $\mu$; the system (6) has an infinite number of solutions, and one would have to proceed to higher-order terms in the standard perturbation expansion in order to determine which of these solutions is relevant.

In our second example, we take $f^{\prime}(s)=\sin (3 \pi s)$, a perturbation that is ineffective at $\lambda=\lambda_{1}\left(\left\langle f^{\prime}, u_{1}\right\rangle=0\right)$ and is effective at $\lambda=\lambda_{2}\left(\left\langle f^{\prime}, u_{2}\right\rangle=-0.67\right)$. In Figure 6 , we show the graphs of $\theta(s)$ for $\lambda=\lambda_{1}, \lambda_{2}$ and $\varepsilon=\frac{1}{4}, \frac{1}{2}$, and 1 .

Our theoretical prediction for the behavior at $\lambda=\lambda_{2}$ comes from Theorem 2 (since $f^{\prime}$ is effective). Using the formulas in that theorem, we compute $\lambda_{2}=$ 80.7629 and $C=0.05557$. Thus, our predicted behavior at $\lambda=\lambda_{2}$ is

$$
\theta(s) \approx-0.05557 \varepsilon^{1 / 3}(2 \cos (8.987 s)-2+8.987 \sin (8.987 s)), \quad \mu \approx 8.976 \varepsilon^{1 / 3} .
$$



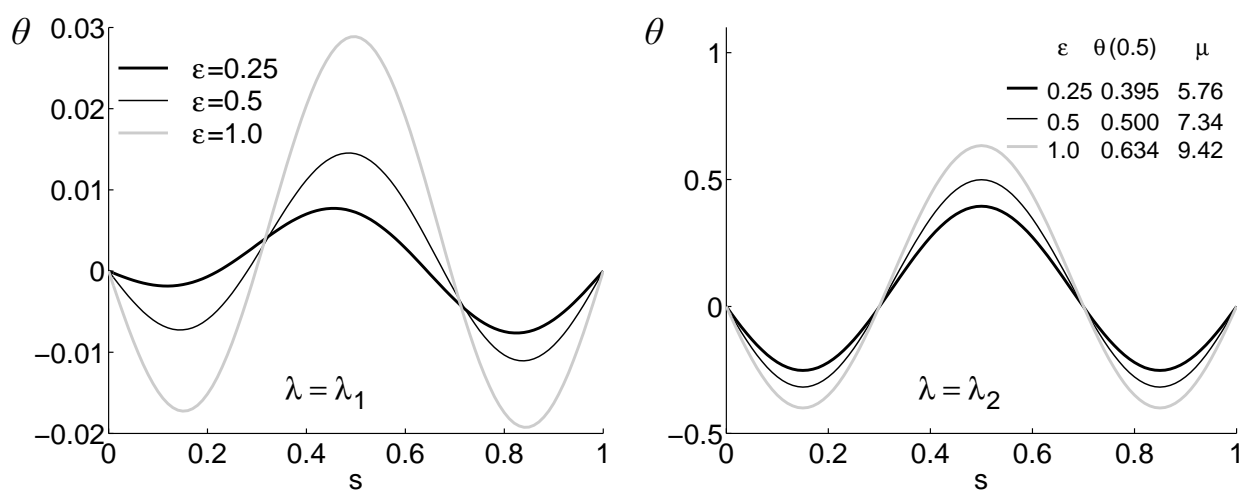

Figure 6. Graphs of $\theta(s)$ for $f^{\prime}(s)=\sin (3 \pi s), \lambda=\lambda_{1}, \lambda_{2}$ and $\varepsilon=\frac{1}{4}, \frac{1}{2}, 1$. For $\lambda=\lambda_{1}$, the dependence of $\theta$ on $\varepsilon$ appears to be approximately $O(\varepsilon)$, in line with the fact that $f^{\prime}$ is ineffective at $\lambda_{1}$. For $\lambda=\lambda_{2}$, the dependence of $\theta$ on $\varepsilon$ appears to be larger than $O(\varepsilon)$, in line with the $O\left(\varepsilon^{1 / 3}\right)$ prediction of the theory. The values of $\theta\left(\frac{1}{2}\right)$ and $\mu$ are also reported.

The shape of the actual solution $\theta(s)$ in Figure 6 matches the predicted functional form, and the scaling with $\varepsilon$ is clearly larger than $O(\varepsilon)$. Furthermore, from the table inset in the figure, we see that both $\theta\left(\frac{1}{2}\right)$ (which from our theoretical formula should equal $0.623 \varepsilon^{1 / 3}$ ) and $\mu$ are close matches with our predicted formulas.

Computational impact. Apart from an interest in understanding on a theoretical level how a shape perturbation affects the bifurcation diagram for buckling, we were also motivated by a pragmatic concern: to what extent ineffective perturbations would interfere with the design of an automated algorithm to compute bifurcation diagrams for a given intrinsic shape. The sharp corners in parts (b), (c) and (d) of Figure 4 suggest potential computational challenges, and we explored that question more concretely with the following numerical study.

We generated intrinsic shapes in three different categories (random, nearly ineffective, and ineffective) as follows. Let $f_{1}(s), f_{2}(s), f_{3}(s), f_{4}(s)$ be the GramSchmidt orthonormal basis (in $\left.L^{2}([0,1])\right)$ generated by the functions $\left\{s, s^{2}, s^{3}, s^{4}\right\}$ :

$$
\begin{array}{ll}
f_{1}(s)=\sqrt{3} s, & f_{3}(s)=15 \sqrt{7} s^{3}-20 \sqrt{7} s^{2}+6 \sqrt{7} s, \\
f_{2}(s)=4 \sqrt{5} s^{2}-3 \sqrt{5} s, & f_{4}(s)=168 s^{4}-315 s^{3}+180 s^{2}-30 s .
\end{array}
$$

The intrinsic shape function $f^{\prime}(s)$ is defined as a linear combination

$$
c_{1} f_{1}(s)+c_{2} f_{2}(s)+c_{3} f_{3}(s)+c_{4} f_{4}(s)
$$


of these basis functions, the coefficients $c_{i}$ being chosen according to different rules for the three cases. For a random perturbation, we choose four independent random numbers $x_{1}, x_{2}, x_{3}, x_{4}$ from a normal distribution with mean 0 and standard deviation 1 , and then define

$$
c_{j}=x_{j} / \sqrt{\left(x_{1}\right)^{2}+\left(x_{2}\right)^{2}+\left(x_{3}\right)^{2}+\left(x_{4}\right)^{2}} .
$$

For an ineffective perturbation, we take $x_{1}, x_{2}, x_{3}$ as above, but then choose $x_{4}$ such that $f^{\prime}(s)$ is ineffective, according to the $n=1$ case of (7), and then normalize to define the $c_{j}$ as in the random case. For a nearly ineffective perturbation, we generate $x_{1}, x_{2}, x_{3}, x_{4}$ as in the ineffective case, but then add to $x_{4}$ a random number with normal distribution with mean 0 and standard deviation 0.01 , before normalizing to define the $c_{j}$ as in the random case.

Given each intrinsic shape, we performed a parameter continuation computation in AUTO97 to attempt to compute the first branch of buckled configurations for that intrinsic shape. We began the computation with $\lambda=28$, zero intrinsic shape, and a straight rod configuration. Then we turned on the intrinsic shape via parameter continuation, by multiplying the intrinsic shape function $f^{\prime}(s)$ by a parameter $\mu$ that was slowly increased from 0 (no intrinsic shape) to 1 (intrinsic shape determined by $f^{\prime}$ ). Then we increased $\lambda$ to a target maximum value of 50. A successful computation would follow the bend of the branch of solutions, with significant rod buckling occurring around $\lambda=4 \pi^{2}$ and continuing until $\lambda$ reaches 50; see part (a) of Figure 4. However, in cases where a sharp corner exists near $\lambda=4 \pi^{2}$, as in the other parts of Figure 4, the computation could jump branches and end at a nearly straight configuration at $\lambda=50$.

To assess in an automated way the success of this computation, we did a third parameter continuation step that decreased $\mu$ from 1 back down to 0 . Thus, successful runs end at the $\lambda=50$ point on the first bifurcating branch for the intrinsicallystraight rod (that is, Figure 3), while unsuccessful runs end with the rod completely extended. Inspection of the value of $y(1)$ at the end of the third parameter continuation step allowed easy distinction of these two cases.

Results of these computations are shown in Table 1. The body of the table shows the percentage of successful runs out of a total of 300 attempts. To give a sense of variability of these results, we report in parentheses the corresponding standard deviation for a binomial random variable with $N=300$ and $p$ taken as the observed percentage of successes: $\sigma=\sqrt{p(1-p) / N}$. (For results reported as $100 \%$ (or $0 \%$ ), all (or none) of the 300 computations were successful, and thus no meaningful estimate of $\sigma$ can be provided).

AUTO97 allows a variety of parameter-stepping algorithms, and Table 1 shows the results for six different approaches: three with fixed step size and three with variable step size. The step sizes $\Delta \tau$ are in terms of a pseudoarc-length that is a 


\begin{tabular}{|c|c|c|c|}
\hline $\begin{array}{c}\text { continuation } \\
\text { method }\end{array}$ & $\begin{array}{c}\text { random } \\
\text { shape }\end{array}$ & $\begin{array}{c}\text { nearly ineffective } \\
\text { shape }\end{array}$ & $\begin{array}{c}\text { ineffective } \\
\text { shape }\end{array}$ \\
\hline$\Delta \tau=0.02$ & $100 \%$ & $41 \%( \pm 3 \%)$ & $0 \%$ \\
$\Delta \tau=0.04$ & $100 \%$ & $16 \%( \pm 2 \%)$ & $0 \%$ \\
$\Delta \tau=0.1$ & $100 \%$ & $0.7 \%( \pm 0.5 \%)$ & $0 \%$ \\
\hline $0.002 \leq \Delta \tau \leq 0.2$ & $99.7 \%( \pm 0.3 \%)$ & $7 \%( \pm 2 \%)$ & $5 \%( \pm 1 \%)$ \\
$0.004 \leq \Delta \tau \leq 0.4$ & $99.7 \%( \pm 0.3 \%)$ & $21 \%( \pm 2 \%)$ & $5 \%( \pm 1 \%)$ \\
$0.01 \leq \Delta \tau \leq 1$ & $98.7 \%( \pm 0.7 \%)$ & $3 \%( \pm 0.9 \%)$ & $0 \%$ \\
\hline
\end{tabular}

Table 1. Percentage of successful computations of the first branch of buckled configurations for different intrinsic shapes: those that are ineffective (in the sense of Section 3), those that are nearly ineffective (small perturbations of exactly ineffective shapes), and randomly chosen shapes (see text for detailed descriptions of the three cases). Each row represents one step-size strategy within the AUTO97 parameter continuation algorithm.

combination of the change in the parameter value $\lambda$ and the change in the solution vector of the discretized Euler-Lagrange equations (1); this approach allows the traversing of "folds" in the bifurcation diagram where $\Delta \lambda=0$. Thus, one can informally think of the change in the parameter $\lambda$ in each step as being some fraction of $\Delta \tau$ (though what that fraction is will vary according to the change in the solution vector at that point on the branch). For the variable step-size computations, the step size $\Delta \tau$ is allowed to varying over two orders of magnitude, with an initial value in the middle (for example, $(\Delta \tau)_{\text {init }}=0.02$ with $0.002 \leq \Delta \tau \leq 0.2$ throughout the computation in row 4 of Table 1). AUTO97 adjusts the step size with each step according to the convergence properties of the previous step, striving to take smaller steps when the convergence is more difficult.

Random and ineffective shapes behave radically differently for the range of step sizes shown here, and even the nearly ineffective shapes show a relatively high rate of computational failure, suggesting that this phenomenon will be met in practice for some shapes (despite the fact that the set of precisely ineffective shapes is measure zero). As would be expected, for fixed step sizes, smaller ones are more successful, though of course at the cost of computation time. (Even for ineffective perturbations, a sufficiently small step size will yield successful branch tracking, though $\Delta \tau$ needs to be significantly smaller than 0.02). For variable step sizes, the data suggests a more complicated situation, including some behavior in the nearly ineffective column that is not monotonic with step-size bounds. This might be explained by the fact that the automated adjustment in step size presumably 
increases the step size consistently in the early part of the computation when the rod is barely changing, and this increased step size might increase the probability of jumping over a corner in the branch (though the likelihood of this jump might also depend sensitively on the initial point chosen, since that could determine whether the jump happens to straddle the corner). Further study would be needed to fully understand this behavior, but it seems clear at least that the ineffectivity condition derived here is a useful flag for intrinsic shapes that call for a strong decrease in the step size.

\section{References}

[Bolshoy et al. 1991] A. Bolshoy, P. McNamara, R. E. Harrington, and E. N. Trifonov, "Curved DNA without A-A: Experimental estimation of all 16 wedge angles", Proc. Natl. Acad. Sci. USA 88:6 (1991), 2312-2316.

[De Santis et al. 1992] P. De Santis, A. Palleschi, M. Savino, and A. Scipioni, "Theoretical prediction of the gel electrophoretic retardation changers due to point mutations in a tract of Sv40 DNA", Biophys. Chem. 42:2 (1992), 147-152.

[Dixit et al. 2005] S. B. Dixit, D. L. Beveridge, D. A. Case, T. E. C. III, E. Giudice, F. Lankas, R. Lavery, J. H. Maddocks, R. Osman, H. Sklenar, K. M. Thayer, and P. Varnai, "Molecular dynamics simulations of the 136 unique tetranucleotide sequences of DNA oligonucleotides, II: Sequence context effects on the dynamical structures of the 10 unique dinucleotide steps", Biophys. J. 89:6 (2005), 3721-3740.

[Doedel et al. 1991a] E. Doedel, H. B. Keller, and J.-P. Kernévez, "Numerical analysis and control of bifurcation problems, I: Bifurcation in finite dimensions", Internat. J. Bifur. Chaos Appl. Sci. Engrg. 1:3 (1991), 493-520. MR 93c:34001a Zbl 0876.65032

[Doedel et al. 1991b] E. Doedel, H. B. Keller, and J.-P. Kernévez, "Numerical analysis and control of bifurcation problems, II: Bifurcation in infinite dimensions", Internat. J. Bifur. Chaos Appl. Sci. Engrg. 1:4 (1991), 745-772. MR 93c:34001a Zbl 0876.65060

[Glendinning 1994] P. Glendinning, Stability, instability and chaos: an introduction to the theory of nonlinear differential equations, Cambridge University Press, Cambridge, 1994. MR 96e:34017 Zbl 0808.34001

[Goyal et al. 2007] S. Goyal, T. Lillian, S. Blumberg, J.-C. Meiners, E. Meyhöfer, and N. C. Perkins, "Intrinsic curvature of DNA influences LacR-mediated looping", Biophys. J. 93:12 (2007), 43424359 .

[Iooss and Joseph 1980] G. Iooss and D. D. Joseph, Elementary stability and bifurcation theory, Springer, New York, 1980. MR 83e:34003 Zbl 0525.34001

[Kahn and Crothers 1998] J. D. Kahn and D. M. Crothers, "Measurement of the DNA bend angle induced by the catabolite activator protein using Monte Carlo simulation of cyclization kinetics", $J$. Mol. Biol. 276:1 (1998), 287-309.

[Manning et al. 1996] R. S. Manning, J. H. Maddocks, and J. D. Kahn, "A continuum rod model of sequence-dependent DNA structure", J. Chem. Phys. 105:13 (1996), 5626-5646.

[Marko and Siggia 1995] J. Marko and E. D. Siggia, "Stretching DNA", Macromolecules 28:26 (1995), 8759-8770.

[Murdock 1999] J. A. Murdock, Perturbations: Theory and methods, Classics in Applied Mathematics 27, Soc. Industrial Appl. Math., Philadelphia, 1999. MR 2000h:34088 Zbl 0810.34047 
[Olson et al. 1998] W. K. Olson, A. A. Gorin, X. J. Lu, L. M. Hock, and V. B. Zhurkin, "DNA sequence-dependent deformability deduced from protein-DNA crystal complexes", Proc. Natl. Acad. Sci. USA 95:19 (1998), 11163-11168.

[Seol et al. 2007] Y. Seol, J. Li, P. C. Nelson, T. T. Perkins, and M. D. Betterton, "Elasticity of short DNA molecules: Theory and experiment for contour lengths of 0.6-7 $\mu \mathrm{m}$ ", Biophys. J. 93:12 (2007), 4360-4359.

[Shifrin and Adams 2002] T. Shifrin and M. R. Adams, Linear algebra: a geometric approach, W. H. Freeman, New York, 2002.

[Swigon et al. 2006] D. Swigon, B. D. Coleman, and W. K. Olson, "Modeling the Lac repressoroperator assembly, I: The influence of DNA looping on Lac repressor conformation", Proc. Natl. Acad. Sci. USA 103:26 (2006), 9879-9884.

Received: 2009-02-12

kaitpeterson@gmail.com

rmanning@haverford.edu
Accepted: 2009-05-02

Mathematics Department, Haverford College, 370 Lancaster Ave., Haverford, PA 19041, United States Mathematics Department, Haverford College, 370 Lancaster Ave., Haverford, PA 19041, United States 